\title{
Calculation of seismic regime parameters on the basis of a prob- abilistic model of Kamchatka earthquake catalogue
}

\author{
Vadim Bogdanov ${ }^{1, \star}$ and Aleksey Pavlov ${ }^{1, \star \star}$ \\ ${ }^{1}$ Institute of Cosmophysical Research and Radio Wave Propagation FEB RAS, Paratunka, Russia
}

\begin{abstract}
In the present paper on the basis of a probabilistic interpretation of the earthquake repetition law, simple formulas were obtained which allow us from the known number of earthquake events in a representative interval of energy class (magnitude) to estimate the number of seismic events falling within an unrepresentative interval and the expected time of an earthquake with a magnitude exceeding the maximum registered values for the period of instrumental observations.
\end{abstract}

\section{Introduction}

The character of Kamchatka seismicity is determined by interaction of several lithospheric plates: Pacific, Okhotsk, Eurasian, North-American and Bering. The majority of earthquakes and the strongest events are associated with Pacific plate subduction. This process is accompanied by tectonic stress accumulation and relaxation and manifests in a large number of earthquakes, the hypocenters of which are associated with the descending oceanic plate. Earthquakes occurring in Kamchatka have different tectonic nature [1]. Figure 1 illustrates six tectonic-geographical zones (structures) of Kamchatka region which have significantly different characteristics of seismicity. $S_{1}, S_{2}, S_{3}$ and $S_{4}$ zones have complicated three-dimensional configuration and sloping edges in depth. $S_{5}$ and $S_{6}$ zones do not contain deep earthquakes and have vertical edges.

On the basis of a probabilistic approach proposed in [2,3], it is possible to analyze the seismic regime for tectonic-geographical zones in Fig. 1. On the basis of probabilistic interpretation of earthquake repetition law graph suggested in [2] and considered in detail in [3, 4], it is possible to obtain simple relations allowing:

- from the known number of earthquakes in a representative energy class (magnitude) to calculate the number of events falling within a unrepresentative interval;

- on the considered area to estimate the expected time of an earthquake with magnitude exceeding maximum registered values for the instrumentation period of observations.

Earthquake repetition law in a probabilistic representation for energy class $K$ distribution density of earthquake events has the following form [2]

$$
f\left(K^{1,2}\right)=f\left(K_{0}^{1,2}\right) \cdot 10^{-\gamma_{1,2}\left(K^{1,2}-K_{0}^{1,2}\right)}
$$

\footnotetext{
${ }_{\star}^{\star}$ e-mail: vbogd@ikir.ru

$\star \star$ e-mail: pavlov@ikir.ru
} 


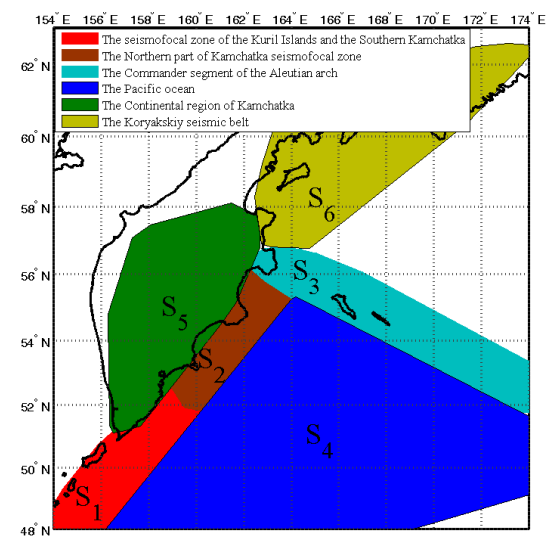

Figure 1. Surface-section of seismicity zones for Kamchatka region

or in a double logarithmic scale the dependence (1) may be presented as

$$
\lg f\left(K^{1,2}\right)=\lg f\left(K_{0}^{1,2}\right)-\gamma_{1,2}\left(K^{1,2}-K_{0}^{1,2}\right)
$$

Expressions (1) and (2) are written for the following ranges of changing of $K: 9.5 \leq K_{1}<12.5$ with $\gamma_{1}\left(K_{0}^{1}=9.5\right)$ and $12.5 \leq K_{2}<15.5$ with $\gamma_{2}\left(K_{0}^{2}=12.5\right)$ and $f\left(K_{0}\right)$ denotes initial distribution density calculated in the middle of the interval $K_{0} \pm \Delta K / 2$.

\section{Calculation of unrepresentative energy class event number}

It was shown by the calculations on the example of Kamchatka earthquake catalogue [3], that it does not depend on the fact if a catalogue is cleared from aftershocks or not, for the fixed period of time $T$ and a given initial interval $K_{0} \pm \Delta K / 2$, the distribution densities $f(K)$, determined by (1), almost do not depend on the considered area where epicenters of events are located. Spread of values appears only in the result of different number of events falling within the considered area. The larger is the number of events, the more stable is the distribution $f(K)$. It is achieved either by the increase of an area for a given time period $T$ or by the increase of a period itself for a fixed area. Table 1 shows the probability values for the earthquakes to fall within the interval of the energy class $K$, calculated for the period $T$ from 01.01.1962 to 01.01.2008 and averaged for $S_{1}, S_{2}, \ldots, S_{6}$ zones for the uncleared catalogue of Kamchatka earthquakes [5].

For different initial $K_{0}$ the following values $P\left(K_{0}\right)$ were obtained when $\Delta K=1$ (Table 1): $P\left(K_{0}=\right.$ $9.5 \pm 0.5)=0.658, P\left(K_{0}=10.5 \pm 0.5\right)=0.692, P\left(K_{0}=11.5 \pm 0.5\right)=0.713, P\left(K_{0}=12.5 \pm 0.5\right)=$ 0.716. The average value of the probability $P_{a v}\left(K_{0}\right)$ for different initial $K_{0}$ is equal to 0.6947 .

Figure 2 shows the dependences (2) for different $K_{0}$, equal to $9.5,10.5,11.5$ and 12.5 , calculated for the tectonic-geographic $S_{2}$ zone. On the basis of the dependence (2) the values of incline angles $\gamma_{1}$ and $\gamma_{2}$ of the earthquake repetition law were calculated (Table 2) for two ranges of the energy class $9 \leq K<13$ and $12 \leq K<16$, correspondingly. The values of $\gamma$ (Table 2) presented in the paper [1] and the values of $\gamma_{1}$ calculated on the basis of probability distributions almost coincide.

Comparing different values of probabilities showed in Table 1, one may note that with the increase of initial $K_{0}$ the values of the obtained probabilities have the tendency of gradual increase. It is 
Table 1. Averaged distributions of probabilities for different initial values $K_{0}$

\begin{tabular}{|l|l|l|l|l|}
\hline Intervals of & Average $P\left(K_{i}\right)$ & Average $P\left(K_{i}\right)$ & Average $P\left(K_{i}\right)$ & Average $P\left(K_{i}\right)$ \\
energy class & for the areas of \\
$K_{i}$ & $\begin{array}{l}S_{1}, S_{2}, \ldots, S_{6}, \\
\text { for the areas of } \\
S_{1}, S_{2}, \ldots, S_{6}, \\
K \geq 10\end{array}$ & $\begin{array}{l}S_{1}, S_{2}, \ldots, S_{6}, \\
K \geq 11\end{array}$ & $\begin{array}{l}S_{1}, S_{2}, \ldots, S_{6}, \\
K \geq 12\end{array}$ \\
\hline $9.5 \pm 0.5$ & 0.658 & & & \\
\hline $10.5 \pm 0.5$ & 0.234 & 0.692 & & \\
\hline $11.5 \pm 0.5$ & 0.076 & 0.218 & 0.713 & \\
\hline $12.5 \pm 0.5$ & 0.023 & 0.064 & 0.205 & 0.716 \\
\hline $13.5 \pm 0.5$ & 0.007 & 0.019 & 0.059 & 0.208 \\
\hline $14.5 \pm 0.5$ & 0.0017 & 0.005 & 0.017 & 0.0602 \\
\hline $15.5 \pm 0.5$ & 0.00072 & 0.002 & 0.00523 & 0.016 \\
\hline
\end{tabular}

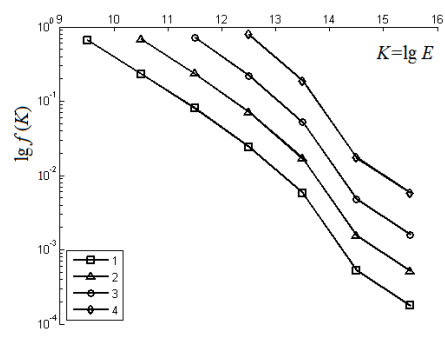

Figure 2. $f(K)$ dependence in a double logarithmic scale (relation (2)) for $S_{2}$ zone for different initial values $K_{0}$ : 1) $K_{0}=9.5 \pm 0.5$, 2) $K_{0}=10.5 \pm 0.5$, 3) $K_{0}=11.5 \pm 0.5$, 4) $K_{0}=12.5 \pm 0.5$.

Table 2. Inclination angle values of the graph of earthquake repetition law

\begin{tabular}{|c|c|c|c|c|c|c|}
\hline & $S_{1}$ & $S_{2}$ & $S_{3}$ & $S_{4}$ & $S_{5}$ & $S_{6}$ \\
\hline$\gamma, 9 \leq K<13$ & -0.5 & -0.43 & -0.45 & -0.7 & -0.53 & - \\
\hline$\gamma_{1}, 9 \leq K<13$ & -0.476 & -0.425 & -0.456 & -0.623 & -0.589 & -0.434 \\
\hline$\gamma_{2}, 12 \leq K<16$ & -0.745 & -0.726 & -0.595 & - & - & -0.309 \\
\hline
\end{tabular}

explained by the fact that with the decrease of $K_{0}$, some difficulties on registration of events appear. They are determined by unbranched network of seismic stations, external disturbing factors and so on, which eventually result in the gaps of occurred earthquakes. Thus, the graph fall of the repetition law for small values of $K$, and, correspondingly, the probability decrease occur due to the decrease of the registered number of events falling within the unrepresentative region of energy class values. On the other side, with the increase of initial value of the complete group of real events decreases and the part of events in the initial chosen interval increases and the probability in frequency presentation grows.

Calculation of distribution density on the basis of real registered events with energy less than a representative one and its comparison with $f\left(K_{i}\right)$, calculated on the basis of the relation (1), give disagreement. Thus, the task appears to obtain a formula for calculation of event number in energy class unrepresentative interval on the basis of known event number and, consequently, distribution density in representative class. Hence, if for period $T$ and for area $S$ within the energy class representative interval $K_{0} \pm 0.5, n_{0}(\Delta K)$ earthquakes were registered when their total number is $N_{\Sigma}$, forming an 
event complete group with energy classes from $K_{0} \pm \Delta K$ to $K_{\max } \pm \Delta K$, than the number of events $n_{-1}$, occurred in the next unrepresentative interval with $K_{-1}<K_{0}$, may be determined by the relation (3).

$$
n_{-1}=\frac{N_{\Sigma} \cdot P_{0}\left(K_{0} \pm \Delta K / 2\right)}{1-P_{0}\left(K_{0} \pm \Delta K / 2\right)}
$$

It is assumed that with $K$ value decrease in unrepresentative class region, $\gamma_{1}$ does not change. Now we determine the number of earthquakes which occurred in the northern part of Kamchatka seismofocal zone ( $S_{2}$ zone) for the period $T$ (from 01.01.1962 to 01.01.2008) and falling within the unrepresentative interval of the energy class $8.5 \pm 0.5$. Since the total number of events with $K \geq 9$, which occurred in $S_{2}$ zone for the period $T$, is $N_{\Sigma}=11323$, and the probability to fall within the initial interval is $P(9.5 \pm 0.5)=0.6582$, then according to the recurrent relation (3) we obtain $n_{-1}=21805$. At the same time according to the earthquake catalogue, the total number of registered events for $T=46$ years on the area $S_{2}$ and falling within the interval $8.5 \pm 0.5$ equals 16893. Similarly, on the basis of (3) the number of events was calculated that fall into energy class interval $8.5 \pm 0.5$ for other considered zones $S_{i}$. The calculation results are shown in Table 3.

Table 3. The number of events in an unrepresentative range of energy class

\begin{tabular}{|c|c|c|c|c|c|c|}
\hline Zone & $S_{1}$ & $S_{2}$ & $S_{3}$ & $S_{4}$ & $S_{5}$ & $S_{6}$ \\
\hline$n(8.5 \pm 0.5)$, (catalogue) & 7308 & 11323 & 3177 & 1534 & 2348 & 710 \\
\hline$n(8.5 \pm 0.5)$, (relation (3)) & 10566 & 21805 & 6361 & 3912 & 3698 & 1000 \\
\hline
\end{tabular}

\section{Calculation of the expectance period of a strong earthquake}

The following expression was obtained in the paper [2] on the basis of relation (1)

$$
\lg N=A-b M
$$

where $A=\lg N\left(M_{0}\right)+b M_{0}=\lg \left[10^{b M_{0}} \cdot N\left(M_{0}\right)\right]$ and $b=1.5 \gamma, N\left(M_{0}\right)$ is the number of events falling within the interval $M_{0} \pm \Delta M / 2, M_{0}=\left(K_{0}-4.6\right) / 1.5\left(M_{0}^{1}=3.27\right.$ for $K_{0}=9.5, M_{0}^{2}=5.27$ for $K_{0}=12.5, \Delta M=0.67$ for $\left.\Delta K=1\right)$. The relation between $K$ class and $M$ magnitude for KurilKamchatka earthquakes was specified by $K=4.6+1.5 M$. When there is no enough statistics in the region of high $M$ values, as $\lg N$ becomes negative, and the time of expectance exceeds the period of instrumentation observations, it is possible to estimate this time by relation (4) for magnitude higher values compared to already registered ones for the period of instrumentation observations. We suppose that spatial and energy characteristics of Kamchatka earthquakes for 01.01.1962-01.04.2016 at the area reflect, in average, its seismic regime. Thus, the average number of events, the epicentres of which are located on area $S_{7}$ (Fig. 3), calculated for a long period of observations (several decades, for example), describes quite well the average regime for a longer period. In this case, from the equality of average calculated for the periods of instrumentation observations $T_{\text {ins }}$ and the required period $T_{\text {req }}$ during which one event may occur with maximum magnitude $M_{\max }$, we obtain

$$
T_{\text {req }}=\frac{10^{1.5 \gamma\left(M_{\max }-M_{0}^{2}\right)}}{N\left(M_{0}^{2}\right)} T_{\mathrm{ins}}
$$

According to (5) the expected time for Kamchatka region for three magnitudes $M_{\max }^{1}=8.5$, $M_{\max }^{2}=9.0$ and $M_{\max }^{3}=9.5$ is estimated. The results of data processing from the catalogue for 


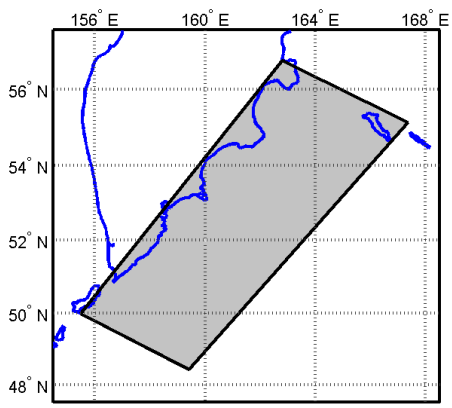

Figure 3. $S_{7}$ zone

Kamchatka region for zone $S_{7}$ for the period of $T_{\text {ins }}=54$ years (from 01.01.1962 to 01.04.2016): $N_{\Sigma}=27117$ is the summary number of events of the catalogue with the energy class within the interval from $9.5 \pm 0.5$ to $15.5 \pm 0.5$ (for the magnitudes from $3.27 \pm 0.335$ to $7.27 \pm 0.335) ; P(5.27 \pm$ $0.335)=0.024$ is the probability for seismic events to fall within the interval $M_{0}^{2} \pm 0.335 ; N\left(M_{0}^{2}\right)=$ $P(5.27 \pm 0.335) \times N_{\Sigma}=0.024 \times 27117=662$ is the number of events registered on $S_{7}$ area for $T_{\text {ins }}$ within the magnitude interval $M_{0}^{2} \pm 0.335 ;|\gamma|=0.64$ is the incline coefficient calculated by the least square method. Calculation on the basis of the given data results in the following values of the expected periods with corresponding magnitudes of events: for $M_{\max }^{1}=8.5$ expected period $T_{\text {req }}^{8.5} \approx 100$ years; for $M_{\text {max }}^{2}=9.0$ expected period $T_{\text {req }}^{9.0} \approx 300$ years; for $M_{\text {max }}^{3}=9.5$ expected period $T_{\text {req }}^{9.5} \approx 960$ years.

\section{Conclusion}

It was shown that for the considered zones of $S_{1}, S_{2}, \ldots, S_{6}$ and different values of the initial interval of the energy class $K_{0} \pm 0.5$, for $K_{0}=9.5,10.5,11.5$ and 12.5 , the probability values $P\left(K_{0} \pm 0.5\right)$ differ little from each other and tend to the average value 0.6947 .

On the basis of recurrence relation (3) the estimation of events which must occur in the unpresentative energy class $K_{0} \pm 0.5$ was calculated for the zones of $S_{1}, S_{2}, \ldots, S_{6}$.

On the basis of relation (5) the estimation of time of a strong earthquake for zone $S_{7}$ was calculated. The periods of expectance of seismic events with magnitudes 8.5, 9.0 and 9.5 were 100, 300 and 960 years, respectively.

\section{References}

[1] V.I. Levina, A.V. Lander, S.V. Miryushkina, A.Yu. Chebrova, Journal of Volcanology and Seismology 7, 37-57 (2013)

[2] V.V. Bogdanov, Doklady Akademii Nauk 408, 393-397 (2006)

[3] V.V. Bogdanov, A.V. Pavlov, A.L. Polyukhova, Journal of Volcanology and Seismology 4, 412$422(2010)$

[4] V.V. Bogdanov, A.V. Pavlov, A.L. Polyukhova, Izvestiya vysshikh uchebnykh zavedeniy. SeveroKavkazskiy region. Seriya: Estestvennye nauki 1, 44-48 (2012)

[5] http://www.emsd.ru/sdis/earthquake/catalogue/catalogue.php

[6] S.A. Fedotov, Energy classification of Kuril-Kamchatka earthquakes and magnitude problem (Nauka, Moscow, 1972) 